\title{
Increase in serum prorenin precedes onset of microalbuminuria in patients with insulin-dependent diabetes mellitus
}

\author{
J.Deinum ${ }^{1}$, B. Rønn ${ }^{2}$, E. Mathiesen ${ }^{2}$, F.H.M.Derkx ${ }^{1}$, W.C.J.Hop ${ }^{3}$, M. A.D.H. Schalekamp ${ }^{1}$ \\ ${ }^{1}$ Department of Internal Medicine I, University Hospital Dijkzigt Rotterdam, The Netherlands \\ ${ }^{2}$ Steno Diabetes Centre, Gentofte, Denmark \\ ${ }^{3}$ Department of Epidemiology and Biostatistics, Erasmus University Rotterdam, The Netherlands
}

\section{Abstract}

Aims/hypothesis. The renin-angiotensin system is possibly involved in the pathogenesis of diabetic nephropathy. The most striking change in renin-angiotensin system components in blood of patients with diabetic nephropathy is an increased prorenin concentration. We investigated prospectively serum concentrations of renin-angiotensin system components and the time course of prorenin increase in normoalbuminuric diabetic patients developing microalbuminuria.

Methods. Patients $(n=199)$ with Type I (insulin-dependent) diabetes mellitus and normoalbuminuria at baseline were prospectively followed for 10 years. The prorenin concentrations and other variables possibly associated with the occurrence of microalbuminuria, were investigated by Cox-regression analysis.

Results. Of the patients 29 developed microalbuminuria. Glycated haemoglobin values were higher at baseline in these patients. Serum prorenin was similar at baseline but rose in the 29 patients before the development of microalbuminuria and was stable in patients with stable albumin excretion. Renin, angiotensinogen and angiotensin converting enzyme serum concentrations were stable in both groups. Prorenin and glycated haemoglobin were independent prognostic factors for the development of microalbuminuria. A prognostic index, based on these variables, was constructed to estimate the relative risk of developing microalbuminuria.

Conclusions/interpretation. Increase in serum prorenin precedes onset of microalbuminuria in normotensive patients with insulin-dependent diabetes mellitus. High concentrations of prorenin in combination with high values of glycated haemoglobin can be used as a predictor of development of microalbuminuria. [Diabetologia (1999) 42: 1006-1010]

Keywords Diabetes mellitus, glycated haemoglobin, microalbuminuria, prognostic index, prorenin.
Progression of microalbuminuria (defined as urinary albumin excretion rate (AER) of 30-300 mg/24h[1]) to overt diabetic nephropathy in patients with Type I (insulin-dependent) diabetes mellitus is retarded by treatment with inhibitors of angiotensin converting enzyme (ACE). Even at the microalbuminuric stage,

Received: 30 December 1998 and in revised form: 1 April 1999

Corresponding author: J. Deinum, Department of Internal Medicine I, Room L253, University Hospital Dijkzigt, Postbus 2040, 3000 CA Rotterdam, The Netherlands

Abbreviations: AER, Albumin excretion rate (urine); ACE, angiotensin converting enzyme; AngI, II, angiotensin I, II; PI, prognostic index. pathologic changes are present in the kidney [2] together with widespread endothelial dysfunction [3] [4]. Microalbuminuria is associated with excess cardiac morbidity [5]. It may therefore be worthwile to identify diabetic patients at risk before the stage of microalbuminuria, to start preventive treatment. Several risk factors for progression to microalbuminuria have been proposed [6,7], such as poor metabolic control, hypertension and high-normal AER.

The most attractive factors for identifying risk are those involved in the pathogenesis of the disease. The favourable response to ACE inhibitors $[8,9]$ suggests that the renin angiotensin system is one which is supported by experimental evidence that angiotensin II (AngII) is an important factor in the pathogenesis 
of diabetic nephropathy [10]. The major change in this system in diabetic patients with microalbuminuria or the ensuing stage of diabetic nephropathy is an increased plasma concentration of prorenin [11, 12]. Prorenin is the inactive precursor of the aspartyl protease renin, which is produced by the kidney. Renin is essential in the proteolytic cascade leading to production of AngII, a key hormone in blood pressure regulation and fluid and electrolyte homeostasis. Data on the change in prorenin concentration over time, in relation to the onset of nephropathy are scarce. In a retrospective longitudinal survey [13] total renin (i.e. prorenin + renin) increases before the onset of microalbuminuria. Here we report on a longitudinal 10-year follow-up study in 199 patients with Type I diabetes mellitus who were normoalbuminuric at the outset. Microalbuminuria developed in 29 patients during follow-up. The aim of this study was to investigate whether the increase in prorenin that has been observed in Type I diabetes mellitus, precedes the development of microalbuminuria.

\section{Subjects and methods}

Patients. Patients with Type I diabetes mellitus $(n=209)$ from the outpatient clinic at the Steno Diabetes Centre (Copenhagen, Denmark) were included between October 1982 and January 1983. Characteristics of the cohort have been reported in detail [6]. Patients gave informed consent to the study and the study was approved by the Ethics Committe of the Steno Diabetes Centre. The investigations were carried out in accordance with the Declaration of Helsinki. All patients fulfilled the following criteria: age between 18 and 50 years, onset of diabetes before the age of 30 years, duration of diabetes 10 to 30 years, diastolic blood pressure less than $100 \mathrm{mmHg}$ and AER less than $30 \mathrm{mg}$ per $24 \mathrm{~h}$ in one urine sample collected at home and remaining normal in at least two out of three samples during the first year of observation. None of the patients received medication, including oral contraceptives, other than insulin. Patients were followed for 10 years. Every 4 months albumin excretion was determined in a 24 -h urine collection. The patients were classified as having microalbuminuria when AER was 30-300 mg/24 h in two out of three urine samples in 2 consecutive years. Blood pressure was measured and ophthtalmoscopy was carried out annually. Blood samples for the measurement of $\mathrm{HbA}_{1 \mathrm{c}}$ were also collected annually. Serum obtained annually was kept at $-20^{\circ} \mathrm{C}$ for later measurement of prorenin, renin, angiotensinogen and angiotensin converting enzyme. Four patients were lost during follow-up and five patients were excluded due to severe diseases (AIDS, cancer, connective tissue disease, hyperthyroidism and chronic pyelonephritis). One patient could not be evaluated because of missing serum samples. Finally 199 patients were evaluated.

During follow-up, 20 patients became pregnant. Serum concentrations of prorenin and renin during pregnancy were excluded from analysis because prorenin is elevated during pregnancy [14]. Of the patients, 13 developing microalbuminuria, also developed hypertension (defined as a blood pressure exceeding 140/95 $\mathrm{mmHg}$ ), 5 before and 8 after the onset of microalbuminuria. Antihypertensive treatment was initiated in only 15 patients of the control group. The antihypertensive drugs that were used were mainly diuretics and calcium chan- nel blockers. Because antihypertensive drugs might influence the serum concentrations of renin and prorenin, only data obtained from patients while not on antihypertensive therapy were included in risk analysis.

Methods. Blood pressure was measured with a standard sphygmomanometer after a 10 -min rest in the sitting position. The disappearance of Korotkoff sounds (phase V) was used to determine diastolic blood pressure. Blood for measurement of prorenin, renin, angiotensinogen and angiotensin converting enzyme was taken after a short rest in the sitting position. Serum was stored at $-20^{\circ} \mathrm{C}$. Retinopathy was assessed by direct ophthalmoscopy by an ophthalmologist. Retinal lesions were categorized as either absent or present, combining background or proliferative retinopathy.

Albumin excretion rate was measured until May 1985 by a radial immunodiffusion technique [15]. Thereafter an enzyme-linked immunoassay was used [16]. Glycated haemoglobin (normal range 4.1-6.4\%) was measured by HPLC (Biorad, Diamat, Richmond, Calif., USA). Serum creatinine was measured by autoanalyser.

Assays of renin and prorenin. Serum renin and prorenin were measured with a commercial kit (Nichols Diagnostics, San Juan Capistrano, Calif., USA), based on an immunoradiometric assay. In this assay renin is measured directly. Total renin, i.e. renin plus prorenin, is measured after enhancement of immunoreactivity of prorenin by preincubation with the renin inhibitor remikiren as described [17]. The difference between total renin and renin is prorenin. The $95 \%$ reference limits are 7.7-54.8 $\mathrm{mU} / 1$ for renin and $88.1-390 \mathrm{mU} / 1$ for prorenin.

Assays of angiotensin converting enzyme and angiotensinogen assays. These assays were done in the 29 patients developing microalbuminuria and in a group of 29 patients remaining normoalbuminuric, matched for age, sex and duration of diabetes. Serum ACE activity was determined with a colorimetric kit (ACEcolor, Fujirebio Inc, Tokyo, Japan), using Gly-His-His as substrate. The concentration of angiotensinogen in serum was determined as the quantity of AngI that is generated after complete hydrolysis of angiotensinogen by recombinant human renin at $\mathrm{pH} 7.4$ and $37^{\circ} \mathrm{C}$ in the presence of inhibitors of AngI degrading enzymes [14]. The AngI was measured by radioimmunoassay [14]. Results are expressed as nmol/l.

Statistical analysis Continuous data were compared using the $t$-test. Serum renin, serum prorenin and urinary albumin excretion were log-transformed before statistical analysis and presented as the geometric mean. The relation of various time-dependent clinical characteristics with the occurrence of microalbuminuria was investigated using Cox-regression analysis [18]. Variables with significant predictive value in univariate analysis were multivariately analysed. Backward elimination was used to determine which factors were independently associated with the occurrence of microalbuminuria. Statistical software used in the analyses was SPSS 7.0 (SPSS Inc. Chicago, Ill., USA).

\section{Results}

Clinical data at entry and during the course of the study. Of the patients, 29 progressed to microalbuminuria during the 10 years of observation and 170 continued with a normal AER. Of the 29 patients that devel- 
Table 1. Clinical data of patients at entry of study

\begin{tabular}{|c|c|c|}
\hline & $\begin{array}{l}\text { Group with } \\
\text { persistent nor- } \\
\text { moalbuminuria } \\
n=170\end{array}$ & $\begin{array}{l}\text { Group devel- } \\
\text { oping microal- } \\
\text { buminuria } \\
n=29\end{array}$ \\
\hline $\operatorname{Sex}(f / m)$ & $74 / 96$ & $13 / 16$ \\
\hline Age (years) & $34 \pm 8$ & $34 \pm 8$ \\
\hline Duration of diabetes (years) & $17 \pm 5$ & $17 \pm 5$ \\
\hline $\operatorname{AER}(\mathrm{mg} / 24 \mathrm{~h})$ & $12(3-29)$ & $17(3-29)^{\mathrm{a}}$ \\
\hline $\mathrm{HbA}_{1 \mathrm{c}}(\%)$ & $8.4 \pm 1.4$ & $9.3 \pm 1.6^{\mathrm{a}}$ \\
\hline $\begin{array}{l}\text { Retinopathy } \\
\text { None } \\
\text { Background } \\
\text { Proliferative }\end{array}$ & $\begin{array}{l}85 \\
80 \\
5\end{array}$ & $\begin{array}{l}10 \\
18 \\
1\end{array}$ \\
\hline $\begin{array}{l}\text { Systolic blood pressure } \\
(\mathrm{mm} \mathrm{Hg})\end{array}$ & $125 \pm 16$ & $128 \pm 13$ \\
\hline $\begin{array}{l}\text { Diastolic blood pressure } \\
(\mathrm{mm} \mathrm{Hg})\end{array}$ & $78 \pm 11$ & $81 \pm 7$ \\
\hline $\operatorname{Renin}(m U / 1)$ & $26.0(23.6-28.7)$ & $\begin{array}{l}29.1 \\
(25.0-33.9)\end{array}$ \\
\hline Prorenin (mU/1) & $270(250-291)$ & $313(254-386)$ \\
\hline Creatinine $(\mu \mathrm{mol} / \mathrm{l})$ & $82 \pm 11$ & $80 \pm 10$ \\
\hline
\end{tabular}

Values are means \pm SD or geometric mean $(95 \% \mathrm{CI})$

${ }^{\mathrm{a}} p<0.05$

oped microalbuminuria, 8 progressed to overt diabetic nephropathy, i.e. an AER exceeding $300 \mathrm{mg} / 24 \mathrm{~h}$. Clinical characteristics of the patients are given in Table 1 . In the patients who later developed microalbuminuria, AER values and $\mathrm{HbA}_{1 \mathrm{c}}$ were higher at outset. Serum prorenin and renin were not statistically significantly different at the time of inclusion. During the 10-year follow-up serum prorenin and renin did not correlate with serum creatinine, $\mathrm{HbA}_{1 \mathrm{c}}$, diastolic or systolic blood pressure. At baseline the concentrations of prorenin were not different in patients with background or proliferative retinopathy, compared with patients without retinopathy (not shown).

Figure 1 shows the levels of prorenin, renin, angiotensinogen and ACE during follow-up. The year of first occurrence of microalbuminuria was designated as year zero. For patients who remained normoalbuminuric throughout follow-up, year zero was chosen to be 1987 , since this was the average year of onset of microalbuminuria in the other group. Data for angiotensinogen and ACE showed stable levels during follow-up. Data in the upper two panels of Figure 1 suggest that serum prorenin and possibly renin increase before microalbuminuria sets in. Univariate Cox-regression analysis of occurrence of microalbuminuria showed statistically significant relations with the concentration of serum prorenin, value of $\mathrm{HbA}_{1 \mathrm{c}}$, and systolic and diastolic blood pressure, and with retinal status. Table 2 summarizes the size of the effect of these variables. No univariate significant relations were found for the duration of diabetes and the serum concentrations of renin and creatinine.
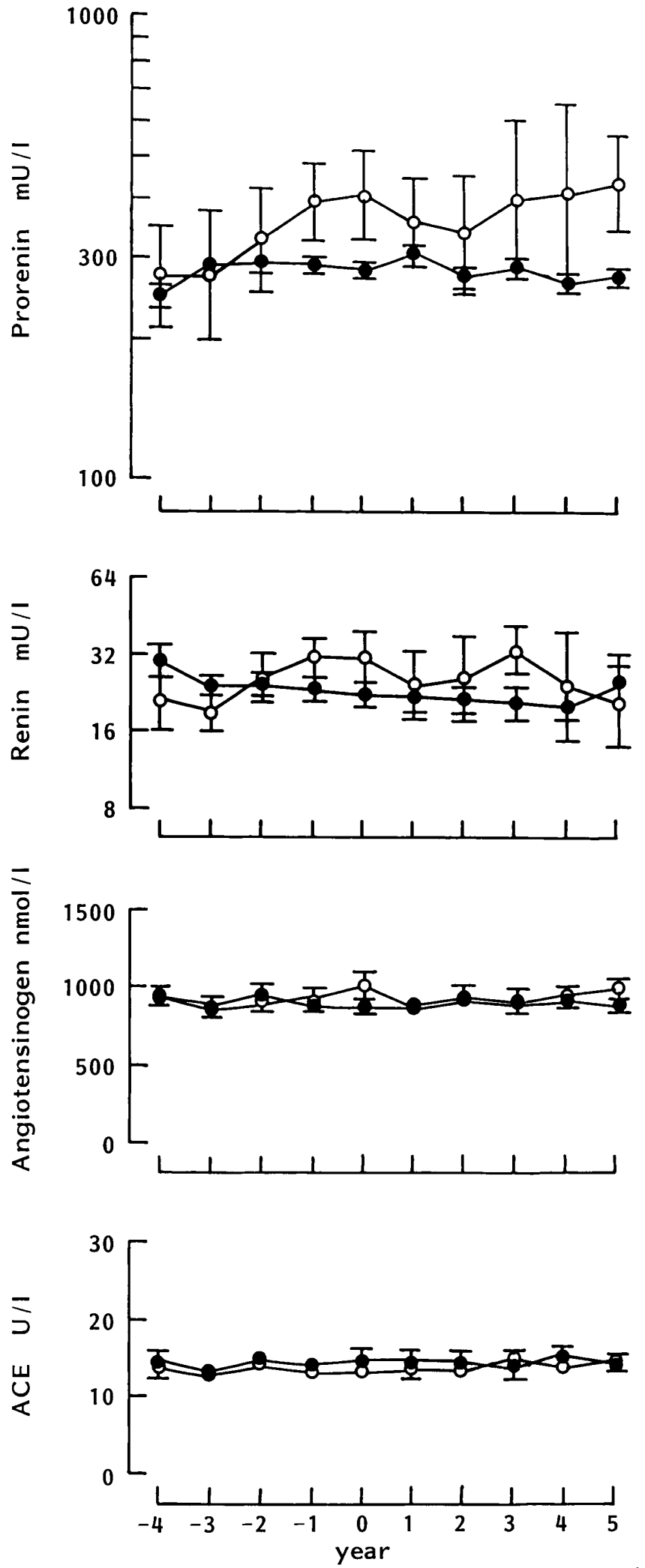

( $0=$ first year of microalbuminuria or 1987)

Fig.1. Serum prorenin, renin, angiotensinogen and ACE in patients remaining normoalbuminuric and $(\bigcirc)$ and in patients developing microalbuminuria $(\mathbf{)})$. Year 0 is the first year of microalbuminuria or 1987 for patients remaining normoalbuminuric. Note the logarithmic scale in the upper two panels. Shown are geometric means $\pm 95 \%$ confidence interval (upper two panels) and means \pm SE (lower two panels) 
Table 2. Results of univariate Cox-regression analysis for the occurrence of microalbuminuria in 199 patients with Type I diabetes mellitus

\begin{tabular}{lll}
\hline Variable & Relative risk $^{\mathrm{a}}$ & $p$ coefficient \\
\hline Prorenin & $2.6^{\mathrm{b}}$ & 0.001 \\
$\mathrm{HbA}_{1 \mathrm{c}}$ & $2.3^{\mathrm{c}}$ & $<0.001$ \\
Systolic BP $(\mathrm{mmHg})$ & $1.3^{\mathrm{d}}$ & 0.019 \\
Diastolic BP $(\mathrm{mmHg})$ & $1.6^{\mathrm{d}}$ & 0.019 \\
Retinopathy & $2.7^{\mathrm{e}}$ & 0.043 \\
\hline
\end{tabular}

${ }^{a}$ As compared to: ${ }^{b}$ twofold lower value, ${ }^{c} 1 \%$ lower value, ${ }^{\mathrm{d}} 10 \mathrm{mmHg}$ lower value, ${ }^{\mathrm{e}}$ no abnormalities (i.e. no retinopathy)

Subjecting the factors prorenin, $\mathrm{HbA}_{1 \mathrm{c}}$, diastolic and systolic blood pressure and the existence of retinopathy to multivariate analysis, we found that the serum concentrations of prorenin and $\mathrm{HbA}_{1 \mathrm{c}}$ values were the most important factors predictive for microalbuminuria. No additional prognostic value was found for diastolic and systolic blood pressure and for retinal status. Furthermore, we observed that the effect of prorenin was statistically significantly affected by the duration of diabetes. Prorenin, but not $\mathrm{HbA}_{1 \mathrm{c}}$, lost its statistical significance as a prognostic factor when the duration of diabetes exceeded 25 years. Table 3 gives the results of the multivariate analysis separately for the duration of diabetes up to 25 years and thereafter. With diabetes mellitus present for less than 25 years the risk of developing microalbuminuria increases 4.7 -fold when prorenin concentrations double. The relative risk associated with a $1 \%$ increase in $\mathrm{HbA}_{1 \mathrm{c}}$ is 2.0.

The results enable a prognostic index (PI) to be constructed for the development of microalbuminuria when diabetes has been present for less than 25 years. This is given by the following formula:

$\mathrm{PI}=1.56 * \log _{2}$ [prorenin $]+0.68 \mathrm{HbA}_{1 \mathrm{c}}$

In Figure 2, the relative risk of developing microalbuminuria is graphed, according to the PI value. A PI value of 18 , corresponding to a prorenin concentration of $400 \mathrm{mU} / 1$ and a $\mathrm{HbA}_{1 \mathrm{c}}$ of $8.9 \%$, was arbitrarily chosen as a reference. If a patient in the study group had a PI that was continuously below 19, corresponding to, for instance, a prorenin concentration of $500 \mathrm{mU} / \mathrm{l}$ with a $\mathrm{HbA}_{1 \mathrm{c}}$ value of $9.7 \%$, the chance of developing microalbuminuria was very small. $\mathrm{Pa}$ -

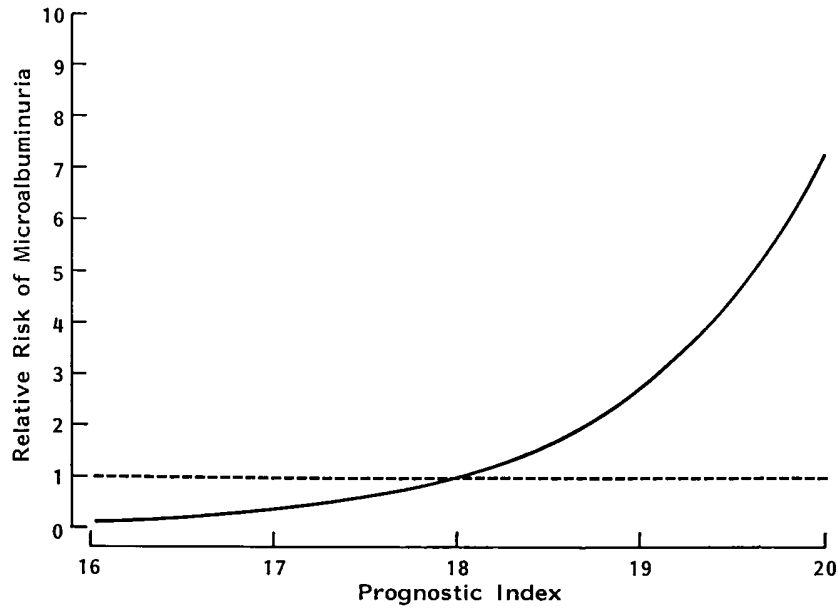

Fig. 2. Relative risk of microalbuminuria in relation to the derived prognostic index PI. The risks are expressed relative to the risk at $\mathrm{PI}=18$

tients scoring a PI exceeding 19, had, however, about $30 \%$ chance of developing microalbuminuria in the next 5 years (data not shown).

\section{Discussion}

This longitudinal, 10-year follow-up study on 199 patients with Type I diabetes mellitus confirms and extends earlier data [13] that indicate that an increase in serum prorenin precedes development of persistent microalbuminuria, the earliest phase in the development of diabetic nephropathy that can be recognized clinically.

The cause of the early rise of prorenin in patients with Type I diabetes mellitus developing nephropathy is not known. Normally, and also under pathological conditions, an increase in prorenin is associated with an increase in renin, the latter being proportionally larger than the former [19]. In the patients with Type I diabetes mellitus the increase in prorenin was not accompanied by an increase in renin, which suggests a derangement of the regulation of the renin-angiotensin system. Whether this derangement plays a causative part in the development of microalbuminuria and nephropathy or is merely a marker of early

Table 3. Relative risk of development of persistent microalbuminuria, determined by prorenin concentrations and glycated haemoglobin values

\begin{tabular}{|c|c|c|c|c|c|c|c|c|}
\hline \multirow[t]{3}{*}{ Variable } & \multicolumn{8}{|c|}{ Duration of diabetes mellitus } \\
\hline & \multicolumn{4}{|l|}{$<25$ years } & \multicolumn{4}{|l|}{$\geq 25$ years } \\
\hline & $\begin{array}{l}\text { Regression } \\
\text { coefficient }\end{array}$ & $\mathrm{RR}$ & $95 \%$ C.I. & $p$ value & $\begin{array}{l}\text { Regression } \\
\text { coefficient }\end{array}$ & $\mathrm{RR}$ & $95 \%$ C.I. & $p$ value \\
\hline $\log _{2}$ (prorenin) & 1.56 & 4.7 & $2.3-9.9$ & $<0.001$ & -1.41 & 0.2 & $0.1-1.2$ & 0.085 \\
\hline $\mathrm{HbA}_{1 \mathrm{c}}(\%)$ & 0.68 & 2.0 & $1.4-2.8$ & $<0.001$ & 1.05 & 2.9 & $1.1-7.7$ & 0.036 \\
\hline
\end{tabular}

$\mathrm{RR}=$ relative risk per doubling of value for prorenin or per per cent increase for $\mathrm{HbA}_{1 \mathrm{c}}, 95 \% \mathrm{CI}=95 \%$ confidence interval 
renal damage, more sensitive than others like albuminuria, is a matter of speculation.

To view our results in a clinical perspective we derived from them a PI, based on $\mathrm{HbA}_{1 \mathrm{c}}$ and serum prorenin. This PI indicates the risk of future microalbuminuria and hence, of diabetic nephropathy. It underlines the importance of good metabolic control. The PI fulfills the following criteria: it has strong predictive power, it is easily determined and it may have pathophysiological relevance in view of the involvement of hyperglycaemia and, with regard to the favourable response to ACE inhibition, of the reninangiotensin system in the development of diabetic nephropathy. Its use must be restricted, however, to normotensive diabetic patients. Moreover, use of drugs, in particular of contraceptive hormones, that considerably affect prorenin concentrations, precludes application of this PI.

A threshold value for PI could be agreed upon above which additional treatment is considered besides optimal therapy for restoring glucose to normal. An obvious choice for supplementary treatment would be ACE inhibition. The EUCLID-study showed that ACE inhibition in normotensive, normoalbuminuric patients had little effect on AER [20]. Use of the proposed PI might have identified a subset of these patients that would have benefited from ACE-inhibition. Obviously, a PI like the one proposed, needs to be validated in other longitudinal studies and work remains to be done to establish international standardization of $\mathrm{HbA}_{1 \mathrm{c}}$ and prorenin assays [21]. Moreover, whether treatment based on this index improves prognosis on a cost-effective basis needs to be determined. Finally, we would like to stress that a reassuring PI value would not be an excuse for inadequate metabolic or blood pressure control.

The model that predicts microalbuminuria included an interaction term for prorenin and duration of diabetes. It is not sure if this statistically obtained variable has a biological meaning. It could mean that microalbuminuria occurring before a duration of 25 years has a different pathogenesis with involvement of the renin-angiotensin system from microalbuminuria occurring very late in the course of Type I diabetes mellitus. This is possibly compatible with reports that the incidence of diabetic nephropathy decreases after 16 years duration of diabetes but has a second smaller peak at about 32 years duration [22].

In conclusion, an increased prorenin concentration in the blood is a herald of microalbuminuria and diabetic nephropathy. Serum prorenin together with glycated haemoglobin can be used to calculate a prognostic index that may be used as a predictor of clinically manifest renal disease in normotensive patients with Type 1 diabetes mellitus.

Acknowledgements. We are grateful to R. de Bruin and J. van Gool for their skillful and dedicated technical assistance.

\section{References}

1. Mogensen CE, Christensen CK, Vittinghus E (1983) The stages in diabetic renal disease with emphasis on the stage of incipient diabetic nephropathy. Diabetes 32 [Suppl 2]: 64-78

2. Østerby R, Anderson AR, Gunderson HJ, Jørgensen HE, Mogensen CE, Parving HH (1984) Quantitative studies of glomerular ultrastructure in juvenile diabetics with incipient nephropathy. Diabetic Nephropathy 3: 95-100

3. Feldt-Rasmussen B (1986) Increased transcapillary escapillary escape rate in type 1 (insulin-dependent) diabetic patients with microalbuminuria. Diabetologia 29: 282-286

4. Jensen T (1991) Albuminuria - a marker of renal and generalized vascular disease in insulin-dependent diabetes mellitus. Dan Med Bull 38: 134-144

5. Deckert T, Yokoyama H, Mathiesen ER et al. (1996) Cohort study of predictive value of urinary albumin excretion for atherosclerotic vascular disease in patients with insulin-dependent diabetes mellitus. BMJ 312: 871-874

6. Mathiesen ER, Rønn B, Storm B, Foght H, Deckert T (1995) The natural course of microalbuminuria in insulin-dependent diabetes: a 10-year prospective study. Diabet Med 12: 482-487

7. Poulsen PL, Ebbehøj E, Hansen KW, Mogensen CE (1977) High normo- or low microalbuminuria: Basis for intervention in insulindependent diabetes mellitus. Kidney Int 52 [Suppl 63]: S15-S18

8. Marre M, Chantellier G, Leblanc H, Guyenne TT, Ménard J, Passa P (1988) Prevention of diabetic nephropathy with enalapril in normotensive diabetics with microalbuminuria. BMJ 297: 1092-1095

9. Mathiesen ER, Hommel E, Giese J, Parving H-H (1991) Efficacy of captopril in postponing nephropathy in normotensive insulin-dependent diabetic patients with microalbuminuria. BMJ 303:210-216

10. Kennefick TM, Anderson S (1997) Role of Angiotensin II in diabetic nephropathy. Semin Nephro 17: 441-447

11. Feldt-Rasmussen B, Mathiesen ER, Deckert T et al. (1987) Central role for sodium in the pathogenesis of blood pressure changes independent of angiotensin, aldosterone and catecholamines in type I (insulin-dependent) diabetes mellitus. Diabetologia 30: 610-617

12. Luetscher JA, Kraemer FB, Wilson DM, Schwartz HC, Bryer-Ash M (1985) Increased plasma inactive renin in diabetes mellitus. N Engl J Med 312: 1412-1417

13. Allen TJ, Cooper ME, Gilbert RE, Winikoff J, Skinner SJ, Jerums G (1996) Serum total renin is increased before microalbuminuria in diabetes. Kidney Int 50: 902-907

14. Derkx FHM, Stuenkel C, Schalekamp MPA, Visser W, Huisveld ICH, Schalekamp MADH (1986) Immunoreactive renin, prorenin, and enzymatically active renin in plasma during pregnancy and in women taking oral contraceptives. J Clin Endocrinol Metab 63: 1008-1015

15. Mancini G, Carbonara AO, Heremans JF (1965) Immunochemical quantitation of antigens by single radial immunodiffusion. Immunochemistry 2: 235-239

16. Feldt-Rasmussen B, Dinesen B, Deckert M (1985) Enzyme-immunoassay: an improved determination of urinary albumin in diabetics with incipient nephropathy. Scand J Clin Lab Invest 45: 539-544

17. Derkx FHM, de Bruin RJA, van Gool JMG (1996) Clinical validation or renin monoclonal antibody-based sandwich assays of renin and prorenin, and use of renin inhibitor to enhance prorenin immunoreactivity. Clin Chem 42: 1051-1063

18. Cox DR (1972) Regression models and life tables. J Royal Statistical Soc 34: $187-220$

19. Deinum J, Schalekamp MADH (1999) Renin and prorenin. In: Oparil S, Weber M (eds) Hypertension. W.B. Saunders Company, Philadelphia, Ch. 7. In press.

20. THE EUCLID STUDY GROUP (1997) Randomized placebocontrolled trial of lisinopril in normotensive patients with insulindependent diabetes and normoalbuminuria or microalbuminuria. Lancet 349:1787-1792

21. Robertson JIS, Nicholls MG (1993) Appendix II. Standardization and Standards. In: Robertson JIS, Nicholls MG (eds) The renin-angiotensin system, vol 2. Gower Medical Publishing, London, pp. P A7-A9

22. Andersen AR, Sandahl Christiansen J, Andersen JK, Kreiner S, Deckert T (1983) diabetic nephropathy in type I (Insulin-dependent) diabetes: An epidemiological study. Diabetologia 25: 496501 